Draft VERSION OCTOBER 9, 2018

Preprint typeset using $\mathrm{IATE}_{\mathrm{E}} \mathrm{X}$ style emulateapj v. 12/16/11

\title{
ACTIVE GALACTIC NUCLEI WITH A LOW-METALLICITY NARROW-LINE REGION
}

\author{
Kota Kawasaki $^{1}$, Tohru NagaO ${ }^{2}$, Yoshiki Toba ${ }^{3}$, Koki Terao ${ }^{1}$, and Kenta Matsuoka ${ }^{4,5,6}$ \\ Draft version October 9, 2018
}

\begin{abstract}
Low-metallicity active galactic nuclei (AGNs) are interesting to study the early phase of the AGN evolution. However most AGNs are chemically matured and accordingly low-metallicity AGNs are extremely rare. One approach to search for low-metallicity AGNs systematically is utilizing the so-called BPT diagram that consists of the $[\mathrm{O}$ III $] \lambda 5007 / \mathrm{H} \beta \lambda 4861$ and $[\mathrm{N}$ II $] \lambda 6584 / \mathrm{H} \alpha \lambda 6563$ flux ratios. Specifically, photoionization models predict that low-metallicity AGNs show a high $[\mathrm{O}$ III $] \lambda 5007 / \mathrm{H} \beta \lambda 4861$ ratio and a relatively low $[\mathrm{N}$ II $] \lambda 6584 / \mathrm{H} \alpha \lambda 6563$ ratio, that corresponds to the location between the sequence of star-forming galaxies and that of usual AGNs on the BPT diagram (hereafter "the BPT valley"). However, other populations of galaxies such as star-forming galaxies and AGNs with a high electron density or a high ionization parameter could be also located in the BPT valley, not only low-metallicity AGNs. In this paper, we examine whether most of emission-line galaxies at the BPT valley are low-metallicity AGNs or not. We select 70 BPT-valley objects from 212,866 emission line galaxies obtained by the Sloan Digital Sky Survey. Among the 70 BPT-valley objects, 43 objects show firm evidence of the AGN activity; i.e., the He II $\lambda 4686$ emission and/or weak but significant broad $\mathrm{H} \alpha$ emission. Our analysis shows that those $43 \mathrm{BPT}$-valley AGNs are not characterized by a very high gas density nor ionization parameter, inferring that at least 43 among 70 BPT-valley objects (i.e., $>60 \%$ ) are low-metallicity AGNs. This suggests that the BPT diagram is an efficient tool to search for low-metallicity AGNs.
\end{abstract}

Keywords: galaxies: abundances — galaxies: active — galaxies: ISM — galaxies: nuclei — galaxies: Seyfert

\section{INTRODUCTION}

The active galactic nucleus (AGN) is one of the most luminous class of objects in the Universe, whose huge radiative energy is released through the mass accretion onto the supermassive black hole (SMBH). The mass of SMBHs $\left(M_{\mathrm{BH}}\right)$ is tightly correlated with the mass or the stellar velocity dispersion of their host galaxies (e.g., Magorrian et al. 1998; Marconi \& Hunt 2003; Kormendy \& Ho 2013; Reines \& Volonteri 2015), implying that SMBHs and galaxies have evolved with closely interacting in each other (the so-called co-evolution of SMBHs and galaxies). However, the physics behind the co-evolution is still unclear. To understand the total picture of the co-evolution, examining the scaling relations for AGNs in the early phase of the co-evolution is an interesting approach since different theoretical models predict different redshift dependences of scaling relations (e.g., Kawakatu et al. 2003; Lamastra et al. 2010). One simple strategy to explore the early phase of the co-evolution is measuring the scaling relations at

kawasaki@cosmos.phys.sci.ehime-u.ac.jp

1 Department of Physics, Graduate School of Science and Engineering, Ehime University, 2-5 Bunkyo-cho, Matsuyama, Ehime 790-8577, Japan

2 Research Center for Space and Cosmic Evolution, Ehime University, 2-5 Bunkyo-cho, Matsuyama, Ehime 790-8577, Japan

3 Academia Sinica Institute of Astronomy and Astrophysics, PO Box 23-141, Taipei 10617, Taiwan

4 Department of Astronomy, Kyoto University, Kitashirakawa- Oiwake-cho, Sakyo-ku, Kyoto 606-8502, Japan

5 Dipartimento di Fisica e Astronomia, Università di Firenze, Via G. Sansone 1, I-50019 Sesto Fiorentino, Italy

6 INAF - Osservatorio Astrofisico di Arcetri, Largo Enrico Fermi 5, I-50125 Firenze, Italy high redshifts, where the typical age of AGNs is much younger than low-redshift AGNs. Many attempts have been made for measuring the scaling relations for highredshift AGNs (e.g., Schramm et al. 2008; Wang et al. 2010; Carniani et al. 2013), and a higher $M_{\mathrm{BH}}$ with respect to the mass or velocity dispersion of host galaxies has been sometimes reported (e.g., Wang et al. 2010). On the other hand, there are some reports claiming that such a possible evolution in the scaling relation is a result of observational bias through the sample selection (e.g., Schulze \& Wisotzki 2011). Measuring the properties of AGN host galaxies at high redshift is generally very challenging, that prevents us from assessing the scaling relations at high redshifts.

Another possible approach to study the early phase of the co-evolution is focusing on young AGNs at low redshifts, where detailed observations are much easier than high redshifts. In this context, low-metallicity (i.e., chemically young) AGNs in the low-redshift Universe are particularly interesting. However, the typical metallicity of AGNs inferred for broad-line regions (BLRs) and narrow-line regions (NLRs) is high $\left(Z \gtrsim 2 Z_{\odot}\right.$; e.g., Nagao et al. 2006b; Matsuoka et al. 2009) and lowmetallicity AGNs are very rare (e.g., Izotov \& Thuan 2008). Groves et al. (2006) proposed a method to search for AGNs with a low-metallicity NLR, that utilizes an optical emission-line diagnostic diagram which consists of the flux ratios of $[\mathrm{N}$ II $] \lambda 6584 / \mathrm{H} \alpha \lambda 6563$ and $[\mathrm{O}$ III $] \lambda 5007 / \mathrm{H} \beta \lambda 4861$. This diagnostic diagram was originally investigated for classifying emission-line galaxies into star-forming galaxies and Seyfert 2 galaxies (BPT diagram, Baldwin et al. 1981). Kewlev et al. (2001, hereafter Ke01) established the "maximum" starburst 
line in the BPT diagram by combining stellar population synthesis models and photoionization models. On the other hand, Kauffmann et al. (2003b, hereafter Ka03) derived empirical classification criteria for star-forming galaxies while Kewley et al. (2006, hereafter Ke06) derived empirical classification criteria for low-ionization nuclear emission-line regions (LINERs; Heckman 1980), using emission-line data taken from the database of Sloan Digital Sky Survey (SDSS; York et al. 2000).

Groves et al. (2006) pointed out that AGNs with a low-metallicity NLRs (i.e., characterized by the solar or sub-solar metallicity) should have a flux ratio of $[\mathrm{O}$ III $] \lambda 5007 / \mathrm{H} \beta \lambda 4861$ as high as usual AGNs $\left(\sim 10^{0.5}-10^{1}\right)$ but have an intermediate flux ratio of $[\mathrm{N}$ II $] \lambda 6584 / \mathrm{H} \alpha \lambda 6563$ between usual AGNs and low-mass (i.e., low-metallicity) star-forming galaxies $(\sim$ $\left.10^{-1}-10^{-0.5}\right)$. This is because the nitrogen relative abundance is in proportion to the metallicity due to its nature as a secondary element (e.g., van Zee et al. 1998). In the BPT diagram, there are only few objects located at the region characterized by a high flux ratio of $[\mathrm{O}$ III $] \lambda 5007 / \mathrm{H} \beta \lambda 4861$ and an intermediate flux ratio of $[\mathrm{N}$ II $] \lambda 6584 / \mathrm{H} \alpha \lambda 6563$ (hereafter "BPT valley"; see Figure 3). Groves et al. (2006) specifically focused on AGNs with a low-mass host galaxy (i.e., $\left.M_{\text {host }}<10^{10} M_{\odot}\right)$, and then they selected low-metallicity AGNs using another diagnostic diagram that consists of $[\mathrm{N}$ II $] \lambda 6584 /[\mathrm{O}$ II $] \lambda 3727$ and $[\mathrm{O}$ III $] \lambda 5007 /[\mathrm{O}$ II $] \lambda 3727$ flux ratios. However, it is not clear whether low-metallicity AGNs should be always found in a sample of AGNs with a low-mass host galaxy. Also, the method adopted by Groves et al. (2006) requires a wide wavelength coverage ( $\left.\lambda_{\text {rest }} \sim 3700-6600 \AA\right)$, that is not convenient for future applications to expand the search of low-metallicity AGNs toward the high-redshift Universe.

Therefore, we focus on BPT-valley selection (requiring a moderately narrow wavelength coverage; $\lambda_{\text {rest }} \sim$ $4800-6600 \AA$ ) to select low-metallicity AGNs without any host-mass cut. However, there is a potentially serious problem in the BPT-valley selection for identifying low-metallicity AGNs. That is, not only low-metallicity AGNs are located in the BPT valley. As Kewley et al. (2013) showed, star-forming galaxies with a very hard radiation field or high-density $\mathrm{H}$ II regions are expected to be seen in the BPT valley (see also, e.g., Shirazi et al. 2014). Also, star-forming galaxies with a high ionization parameter (e.g., Steidel et al. 2014; Havashi et al. 2015), a high nitrogen-to-oxygen abundance ratio (N/O; e.g., Masters et al. 2014; Shapley et al. 2015; Yabe et al. 2015; Kojima et al. 2016), or shocks (e.g., Newman et al. 2014) are also expected to be seen in the BPT valley. Not only star-forming galaxies, AGNs with a high electron density or high ionization parameter (i.e., not characterized by a low metallicity) could be also seen in the BPT valley (e.g., Nagao et al.2001a). Therefore, it is not completely clear whether the BPT-valley objects are really low-metallicity AGNs and whether the BPT diagram is a useful tool to search for low-metallicity AGNs. This problem prevents us from selecting chemically-young AGNs observationally.

In this paper, we investigate the optical spectra of BPT-valley objects for examining whether most of emission-line galaxies at the BPT valley are really low- metallicity AGNs or not. Through this examination, it will be tested whether the optical BPT diagram is an efficient and appropriate method to search for lowmetallicity AGNs. In Section 2, we present our selection procedure of the BPT-valley sample. In Section 3, we show how we identify BPT-valley AGNs to avoid contaminating star-forming galaxies at the BPT valley. In Section 4, we investigate gas properties of the selected BPT-valley AGNs such as electron density and ionization parameter, for examining whether the BPT-valley AGNs are characterized by a low metallicity or not. In Section 5, we disccus physical properties of the BPTvalley AGNs. Section 6 describes the summary of this work.

\section{SAMPLE}

In order to select the BPT-valley objects, we use MaxPlanck-Institute for Astrophysics (MPA)-Johns Hopkins University (JHU) SDSS Data Release 7 (Abazajian et al. 2009) galaxy catalog. The MPA-JHU DR7 catalog of spectral measurements contains various spectral properties such as emission-line fluxes and their errors, based on the analysis for 927,552 objects without showing dominant broad Balmer lines (i.e., star-forming galaxies, composite galaxies, LINERs, and type-2 Seyfert galaxies) in the SDSS DR7. Our sample selection is based on the following procedure (the flow chart of our sample selection process is shown in Figure 11).

First, we select the initial sample according to the following criteria. We require the reliable redshift measurement (i.e., $z_{\text {warning }}=0$ ) and also $z>0.02$. This redshift limit is required to cover $[\mathrm{O}$ II $] \lambda 3727$. This results in 906,761 galaxies. Then we require a signal-tonoise ratio $(\mathrm{S} / \mathrm{N})>3$ for some key emission lines, i.e., $\mathrm{H} \beta \lambda 4861,[\mathrm{O}$ III $] \lambda 5007$, [O I] $\lambda 6300, \mathrm{H} \alpha \lambda 6563$, [N II] $\lambda 6584$ and [S II] $\lambda \lambda 6717,31$ (212,866 galaxies).

Next, we classify these 212,866 galaxies and extract the BPT-valley sample according to the following steps.

1. Using the $\mathrm{Ka03}$ empirical line,

$$
\log \left(\frac{[\mathrm{O} \mathrm{III}]}{\mathrm{H} \beta}\right)>\frac{0.61}{\log ([\mathrm{N} \mathrm{II}] / \mathrm{H} \alpha)-0.05}+1.3
$$

for removing usual star-forming galaxies $(56,217$ galaxies).

2. Using the Ke01 maximum starburst line,

$$
\log \left(\frac{[\mathrm{O} \mathrm{III}]}{\mathrm{H} \beta}\right)>\frac{0.61}{\log ([\mathrm{N} \mathrm{II}] / \mathrm{H} \alpha)-0.47}+1.19,
$$

for removing so-called composite galaxies $(22,865$ galaxies).

3. Using the $\mathrm{Ke} 06$ empirical criterion,

$$
\log \left(\frac{[\mathrm{O} \mathrm{III}]}{\mathrm{H} \beta}\right)>1.36 \log \left(\frac{[\mathrm{O} \mathrm{I}]}{\mathrm{H} \alpha}\right)+1.4,
$$

for obtaining Seyfert sample by removing LINERs (14,253 galaxies).

${ }^{1}$ http://www.mpa-garching.mpg.de/SDSS/ 
4. Adopting the following criterion,

$$
\log \left(\frac{[\mathrm{N} \text { II }]}{\mathrm{H} \alpha}\right)<-0.5
$$

for finally selecting the BPT-valley sample (71 galaxies).

Note that 1 object in the 71 BPT-valley objects was observed twice and duplicated in the final sample, i.e., the final BPT-valley sample consists of 70 objects. The BPT-valley criterion (Equation 4) is determined empirically, by taking account of the frequency distribution of the $[\mathrm{N}$ II $] \lambda 6584 / \mathrm{H} \alpha \lambda 6563$ flux ratio of Seyfert galaxies. Figure 2 shows the $[\mathrm{N} \mathrm{II}] \lambda 6584 / \mathrm{H} \alpha \lambda 6563$ frequency distribution of Seyfert galaxies, where the average and standard deviation of the logarithmic [N II] $\lambda 6584 / \mathrm{H} \alpha \lambda 6563$ flux ratio are -0.058 and 0.145 , respectively. Accordingly, the $3 \sigma$ bounding from the average value is -0.493 , and therefore we adopt the threshold to categorize BPTvalley objects as described by Equation 4, Figure 3 shows the finally selected $70 \mathrm{BPT}$-valley objects in the BPT diagram that consists of $[\mathrm{N}$ II $] \lambda 6584 / \mathrm{H} \alpha \lambda 6563$ versus $[\mathrm{O}$ III $] \lambda 5007 / \mathrm{H} \beta \lambda 4861$. Table 1 shows the basic properties of the selected BPT-valley objects.

\section{SELECTION OF SECURE-AGN SAMPLE}

As described in Section 1, the BPT-valley sample potentially includes star-forming galaxies with special gas properties, not only AGNs. Thus we first select objects showing secure evidence of the AGN from the BPT-valley sample. Specifically, we regard objects showing at least one of the following two features in their SDSS spectra as secure AGNs; (1) a broad $\mathrm{H} \alpha \lambda 6563$ emission, and (2) a He II $\lambda 4686$ emission line. Details of the selection procedure of secure AGNs are given below.

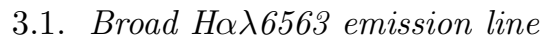

The velocity profile of recombination lines is a powerful tool to examine the presence of AGNs, since star-forming galaxies never show a velocity width wider than $\sim 1000$ $\mathrm{km} \mathrm{s}^{-1}$ in full-width at half maximum (FWHM). Generally the optical spectra of type-1 AGNs show broad permitted lines whose velocity width is $\gtrsim 2000 \mathrm{~km} \mathrm{~s}^{-1}$ emitted from BLRs. The origin of recombination lines with FWHM $\sim 1000-2000 \mathrm{~km} \mathrm{~s}^{-1}$ is not very clear, since such lines may arise at BLRs in so-called narrowline Seyfert 1 galaxies (NLS1s; e.g., Osterbrock \& Pogge 1985) or at NLRs in type-2 AGNs with a relatively large velocity width (such as NGC 1068 and NGC 1275; see, e.g., Heckman et al. 1984; Crenshaw \& Kraemer 2000). However, in either case, the detection of recombination lines with FWHM $>1000 \mathrm{~km} \mathrm{~s}^{-1}$ strongly suggests the presence of AGNs. Therefore we search for the broad H $\alpha \lambda 6563$ component in the optical spectrum of the BPTvalley objects. Here we do not search for the broad component of the $\mathrm{H} \beta \lambda 4861$ emission, since it is intrinsically fainter than that of the $\mathrm{H} \alpha \lambda 6563$ emission and it is sometimes affected significantly by the Fe II multiplet emission (e.g., Vanden Berk et al. 2001).

We use an IRAF routine specfit (Kriss 1994) to find the broad H $\alpha \lambda 6563$ component. Specifically, we fit the SDSS optical spectrum of the BPT-valley objects in the range of $\lambda_{\text {rest }}=6200-6800 \AA$ with and without the

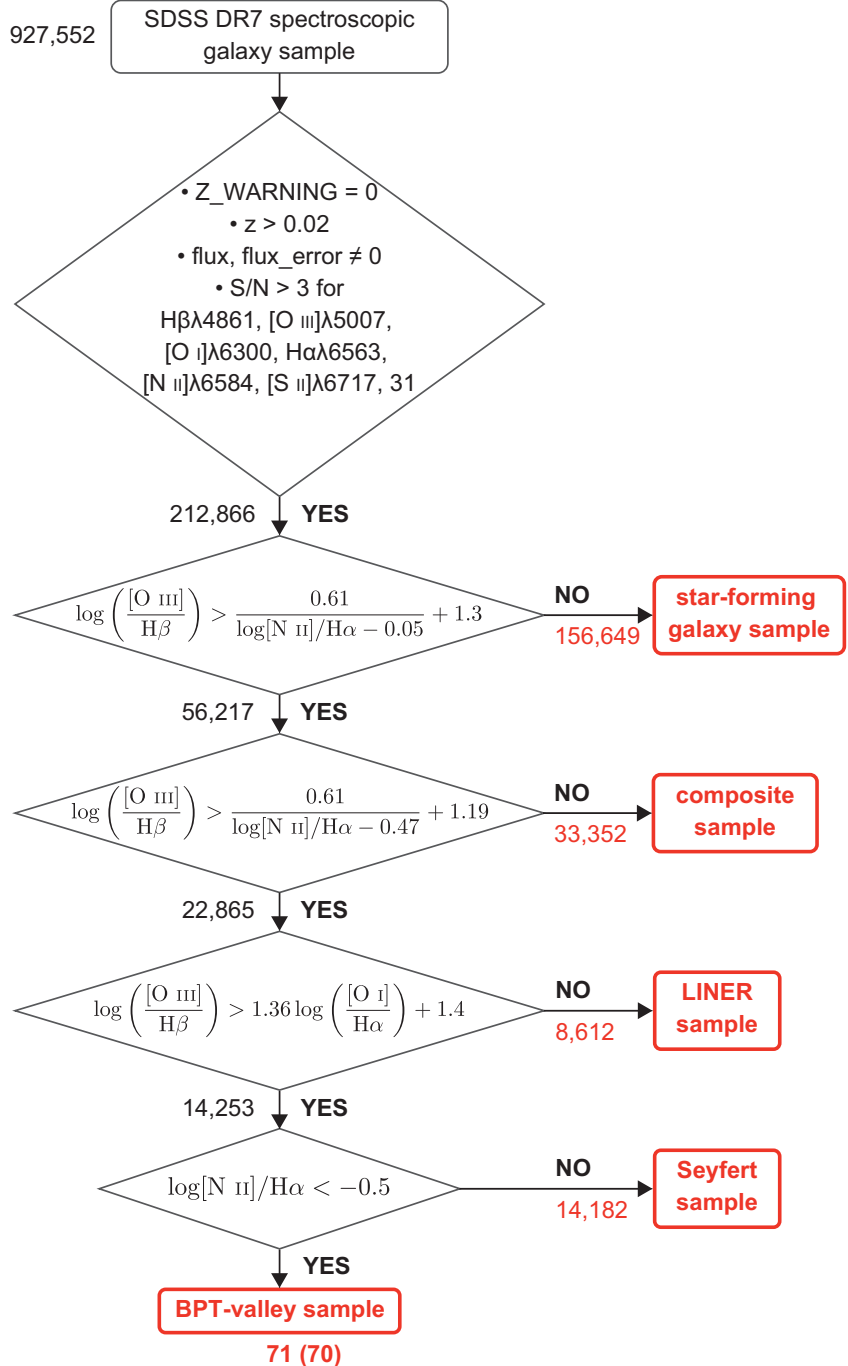

Figure 1. Flow chart of the selection of BPT-valley objects. Numbers shown in the chart denote the numbers of objects at each selection stage. The number shown in the parenthesis denotes the number of objects after removing the duplication.

broad $\mathrm{H} \alpha \lambda 6563$ component, and examine whether the addition of the broad component improves the spectral fit significantly. The details of the fitting procedure are as follows. First, we fit the optical spectrum with a linear continuum component and single-Gaussian emissionline components for $[\mathrm{O} \mathrm{I}] \lambda 6300,[\mathrm{O} \mathrm{I}] \lambda 6363$, [N II] $\lambda 6548$, $\mathrm{H} \alpha \lambda 6563$, [N II] 66584 , [S II] 6717 , and [S II] $\lambda 6731$ (hereafter "nobroad fitting"). Here we assume that the velocity width of all emission lines is the same, and the relative separation of the emission lines is fixed to be the same as that of their laboratory wavelengths. The flux ratios of $[\mathrm{O}$ I] $] 6300$ to $[\mathrm{O} \mathrm{I}] \lambda 6363$ and $[\mathrm{N} \mathrm{II}] \lambda 6584$ to $[\mathrm{N}$ II] $\lambda 6548$ are fixed to be 3.00 and 2.96 respectively (Mendoza 1983), and the flux ratios among the remaining emission lines are kept to be free. Then, we add a broad component for the $\mathrm{H} \alpha \lambda 6563$ emission to the nobroad fit, where the flux, wavelength center and width of this additional component are kept to be free (hereafter "broad fitting"). Here we recognize that the additional broad component significantly improves the fit by the 
Table 1

The BPT-valley sample

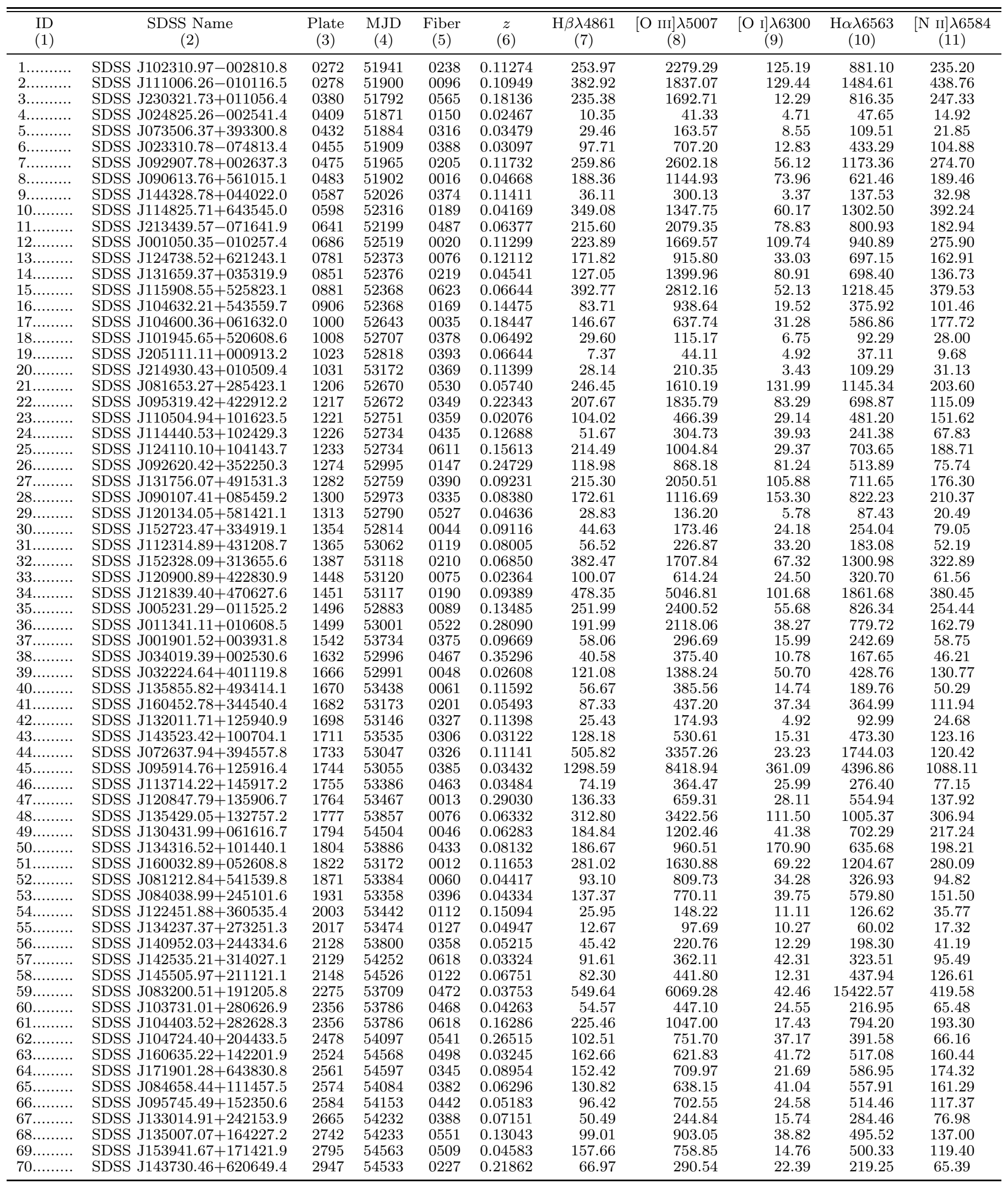

Note. - Col. (1): Identification number assigned in this paper. Col. (2): Object name. Col. (3)-(5): Plate-MJD-Fiber ID in the SDSS observation for analyzed spectra. Col. (6): Redshift measured by the SDSS pipeline. Col. (7)-(11): Emission-line fluxes in units of 10-17 erg $\mathrm{s}^{-1} \mathrm{~cm}^{-2}$. 


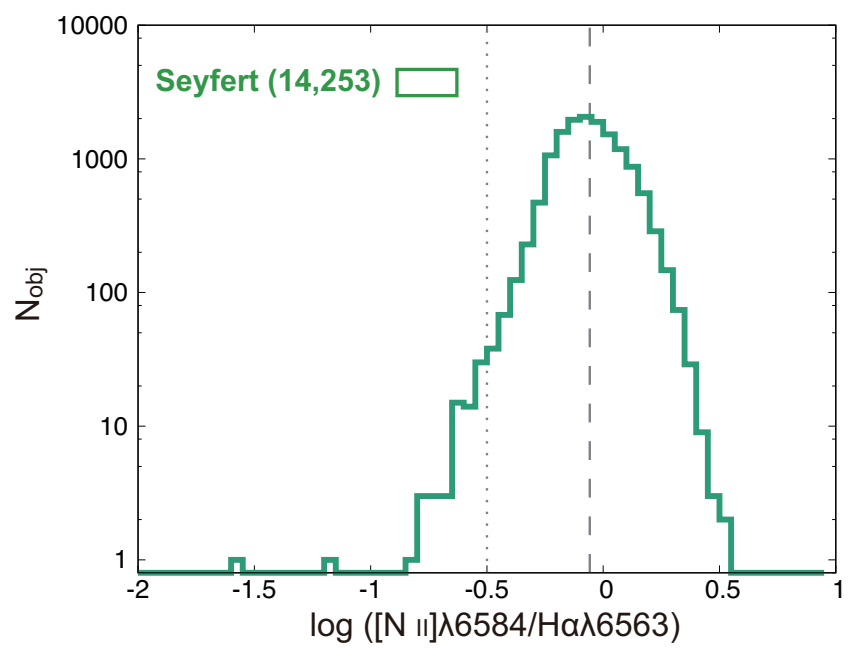

Figure 2. Histogram of the $\left[\begin{array}{ll}\mathrm{N} & \mathrm{II}\end{array}\right] \lambda 6584 / \mathrm{H} \alpha \lambda 6563$ flux ratio of Seyfert galaxies. Dashed line denotes the average of log $\left(\left[\mathrm{N}_{\mathrm{II}}\right] \lambda 6584 / \mathrm{H} \alpha \lambda 6563\right)=-0.058$, while dotted line denotes the threshold of $\log \left(\left[\begin{array}{ll}\mathrm{N} & \mathrm{II}\end{array}\right] \lambda 6584 / \mathrm{H} \alpha \lambda 6563\right)=-0.5$ to select BPTvalley objects.

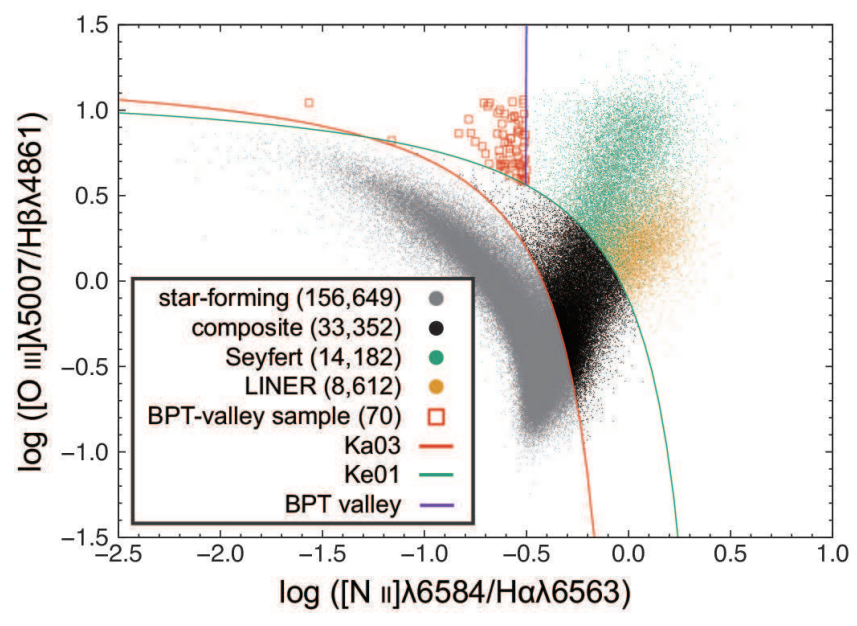

Figure 3. The BPT diagram ([N $\mathrm{II}] \lambda 6584 / \mathrm{H} \alpha \lambda 6563$ versus [O III $] \lambda 5007 / \mathrm{H} \beta \lambda 4861$ ), showing the BPT-valley sample (red square) among the SDSS DR7 emission-line objects. The green solid line is the Ke01 extreme starburst criterion, while the red solid line denotes the criterion for separating star-forming galaxies and composite galaxies (Ka03). The violet solid line is the BPT valley criterion which is defined in this paper. The numbers of various galaxy populations are shown in the parenthesis in the lower-left box.

following criterion:

$$
\frac{\tilde{\chi}_{\text {nobroad }}^{2}-\tilde{\chi}_{\text {broad }}^{2}}{\tilde{\chi}_{\text {nobroad }}^{2}}>0.4,
$$

where $\tilde{\chi}_{\text {nobroad }}^{2}$ and $\tilde{\chi}_{\text {broad }}^{2}$ are the reduced chi-square of the nobroad fitting and broad fitting, respectively. Note that the threshold, 0.4 , is determined empirically, so that the result becomes consistent with the visual inspection. As a result, 13 BPT-valley objects with a broad component are identified from the 70 BPT-valley objects. Figures 4 and 5 show the SDSS spectrum with the best-fit result for the BPT-valley objects with a broad $\mathrm{H} \alpha \lambda 6563$ component. Figure 6 shows an example of objects $(\mathrm{ID}=$ 48) whose fitting result does not satisfy the criterion defined by Equation 5 (for this case, the improvement of the fit is slightly less than the threshold, 0.32). Note that we regard object ID $=8$ as an object with a broad $\mathrm{H} \alpha$ component, though the FWHM of the broad $\mathrm{H} \alpha$ component is less than $1000 \mathrm{~km} \mathrm{~s}^{-1}$ (Figure 7). This is because this object shows $[\mathrm{Fe}$ VII $] \lambda 6087$ and $[\mathrm{Fe} \mathrm{x}] \lambda 6374$ lines, that are seen only when the AGN presents. Note that such high-ionization forbidden emission lines are preferentially seen in type-1 AGNs (e.g., Murayama \& Taniguchi 1998; Nagao et al. 2000). Note that the [Fe VII] $\lambda 6087$ line is seen in 8 objects while [Fe $\mathrm{x}] \lambda 6374$ line is seen in 3 objects (including ID $=8$, note that 2 objects in addition to $\mathrm{ID}=8$ show both $[\mathrm{Fe} \mathrm{VII}] \lambda 6087$ and $[\mathrm{Fe} \mathrm{x}] \lambda 6374)$. The spectral properties of the BPT-valley objects with a broad $\mathrm{H} \alpha \lambda 6583$ component are summarized in Table 2. Only 1 BPT-valley object $(\mathrm{ID}=25)$ shows the broad $\mathrm{H} \beta$ component among the $13 \mathrm{BPT}$-valley objects showing a broad $\mathrm{H} \alpha$ component (see Figure 4).

\subsection{He II $\lambda 4686$ emission line}

The presence of a He II $\lambda 4686$ emission line indicates the existence of the hard ionizing radiation since the ionization potential for $\mathrm{He}^{+}$is $54.4 \mathrm{eV}$. This hard radiation is naturally produced by AGNs. Therefore, the He II $\lambda 4686$ emission line is a good indicator of AGNs. We examine whether the SDSS optical spectrum of the BPT-valley objects show the He II $\lambda 4686$ line by the visual inspection, since the He II $\lambda 4686$ information is not given in the MPA-JHU database. As a result, 38 BPT-valley objects with the He II emission line are identified from the 70 BPT-valley objects. Some of the SDSS spectra of BPT-valley objects with the He II detection are shown in Figures 4, while those without the He II detection are shown in Figure 5 .

\subsection{Classification result of the BPT-valley sample}

The results of the classification of the BPT-valley objects are summarized in Table 3. Among the 70 BPTvalley objects, 8 objects show both broad $\mathrm{H} \alpha$ component and He II emission line, that are now confirmed to be AGNs. There are 5 objects showing the broad $\mathrm{H} \alpha$ component but without He II emission line, that are also regarded as AGNs. The non-detection of the He II line is likely due to insufficient signal-to-noise ratio, since the He II line is very weak. In addition, 30 objects show the He II line but without broad $\mathrm{H} \alpha$ component, that are thought to be typical type-2 AGNs. Here we should mention that the stellar absorption lines (mainly $\mathrm{H} \alpha$ ) are not considered in our fitting procedure. Though the stellar $\mathrm{H} \alpha$ absorption line could impact the narrow component of the $\mathrm{H} \alpha$ emission, the absorption effect is negligible for examining the presence of the broad $\mathrm{H} \alpha$ component. This is because the equivalent width of the detected broad $\mathrm{H} \alpha$ component is higher than $20 \AA$ (the median value of $\mathrm{EW}_{\text {rest }}(\mathrm{H} \alpha)_{\mathrm{b}}$ is $44.74 \AA$, Table 2$)$ while the typical equivalent width of the stellar $\mathrm{H} \alpha$ absorption is $\sim 2-3 \AA$ in nearby galaxies (e.g., Ho et al. 1997). Note that the detected He II line is not caused by Wolf-Rayet stars, because the typical velocity width of the detected He II line is not broad $\left(\lesssim 1000 \mathrm{~km} \mathrm{~s}^{-1}\right)$. Therefore, at least 43 among the BPT-valley objects are regarded as AGNs. There may be some additional AGNs in the remaining 27 objects, possibly owing to insufficient S/N to detect any AGN indicators in their spectra. Instead, 
KAWASAKI ET AL.

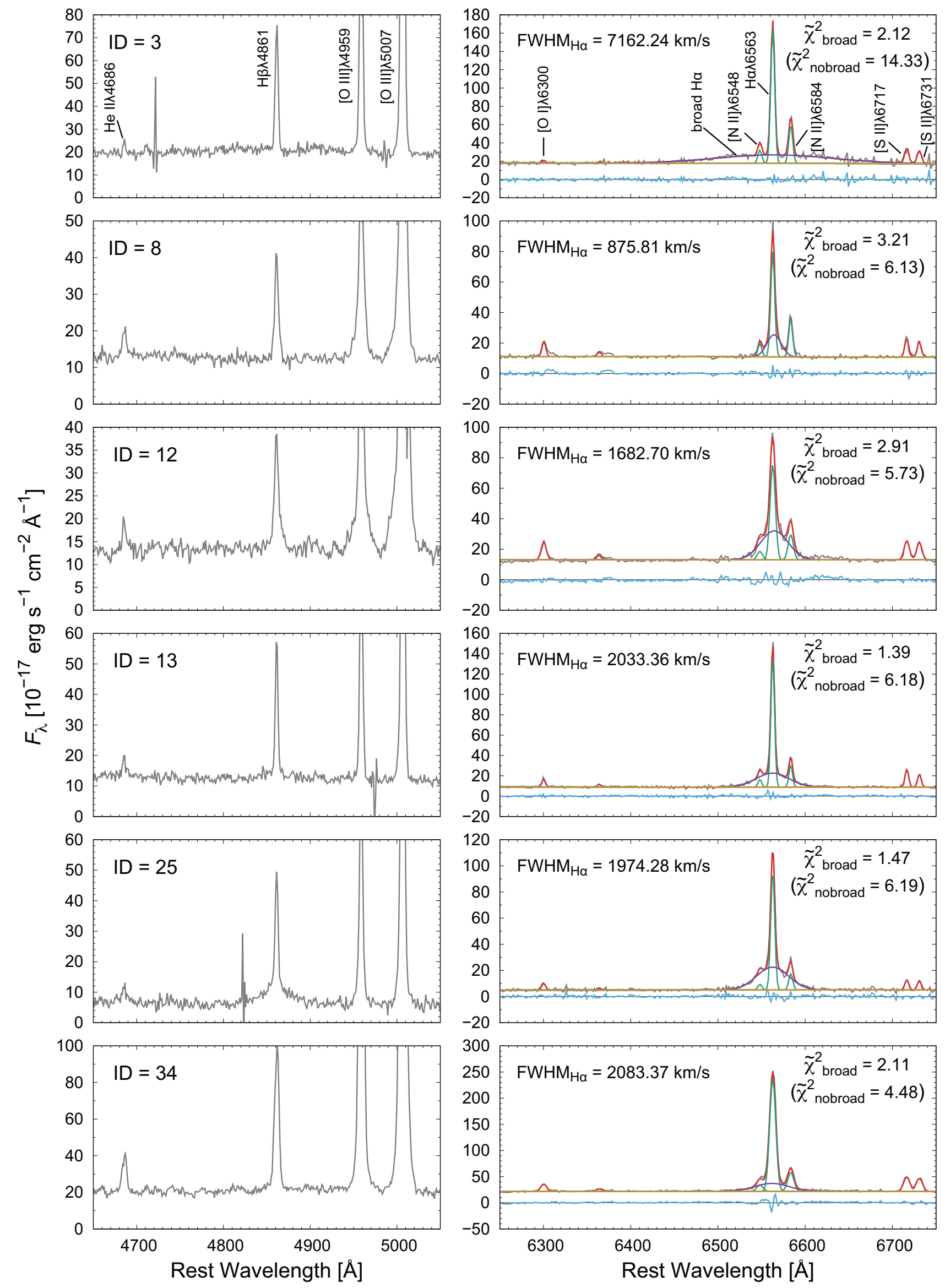

Figure 4. Spectra of the BPT-valley objects showing a broad $\mathrm{H} \alpha$ component and He II emission. Best-fit models are plotted in red, while the narrow-H $\alpha+[\mathrm{N}$ II] Gaussian components, broad $\mathrm{H} \alpha$ component, and continuum are plotted in green, violet, and orange, respectively. Residuals are plotted in blue. Reduced chi-square values are given at the upper-right side in the right panels (the value before adding the broad $\alpha$ component is given in the parenthesis). 


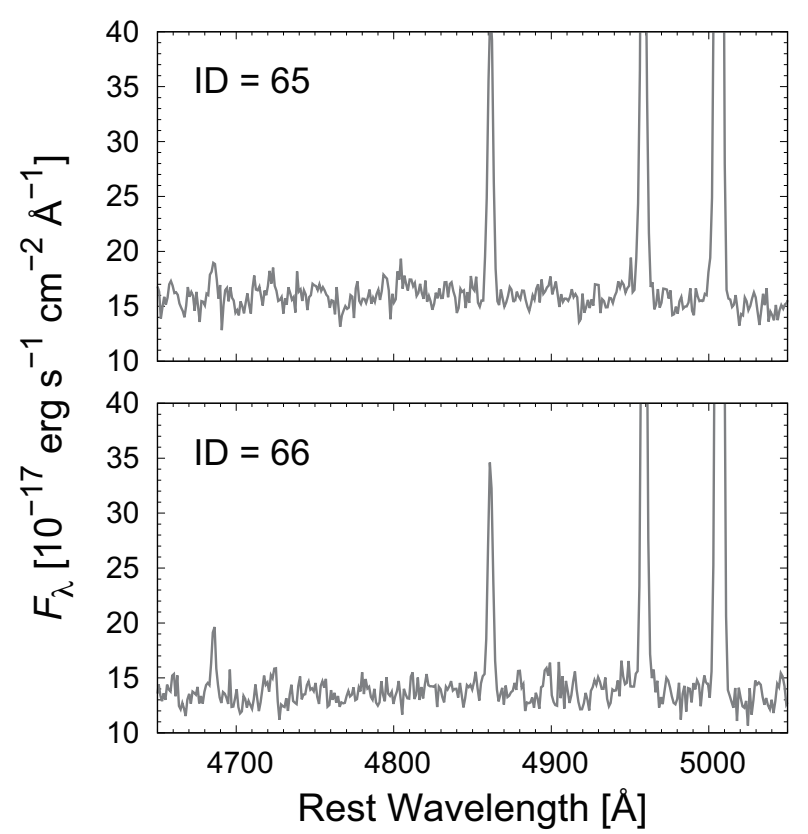

Figure 4. (Continued)

some of those 27 objects could be non-AGNs, i.e., starforming galaxies with a relatively high $\mathrm{N} / \mathrm{O}$ ratio or fast shocks. We do not discuss further about those 27 objects since the main interests of this work are on the BPT-valley AGN sample. Accordingly, we conclude that at least 43 objects of the BPT-valley sample (or $\sim 60 \%$, but probably more) are confirmed to be AGNs.

As described in Section 3.1, at least one of the $[\mathrm{Fe} \mathrm{VII}] \lambda 6087$ and $[\mathrm{Fe} \mathrm{x}] \lambda 6374$ lines are seen in 9 BPTvalley objects. Interestingly, a large fraction of objects showing both the broad $\mathrm{H} \alpha$ component and He II emission show such high-ionization iron lines (5 among 8 objects). On the other hand, objects showing neither the broad $\mathrm{H} \alpha$ component nor He II emission never shows those high-ionization iron lines. Then, a few objects in the remaining two classes show high-ionization iron lines (4 among 35 objects). This may infer that our classification is well tracing the presence of the AGN, but the absence of high-ionization iron lines could be simply due to a low $\mathrm{S} / \mathrm{N}$ ratio of the spectra.

Figure 8 shows how various populations of galaxies classified in this work are populated in the BPT diagram. There are no significant segregation except for two BPT-valley objects whose $[\mathrm{N} \mathrm{II}] \lambda 6584 / \mathrm{H} \alpha \lambda 6563$ flux ratio is very low, $<0.1$. Both of these two galaxies show no broad $\mathrm{H} \alpha$ component nor He II line, which is consistent with the idea that these two objects are not lowmetallicity AGNs but somewhat extreme low-metallicity galaxies, characterized probably by a very high ionization parameter and/or very hard ionization radiation.

\section{SELECTION OF LOW-METALLICITY AGNS}

The 43 BPT-valley objects confirmed to be AGNs are not necessarily low-metallicity AGNs, because AGNs with a very high electron density or very high ionization parameter are also expected to be populated in the BPT valley as mentioned in Section 1. More specifically, the $[\mathrm{N} \mathrm{II}] \lambda 6584$ emission in AGNs with a density higher than the critical density of the $[\mathrm{N} \mathrm{II}] \lambda 6584$ transi-

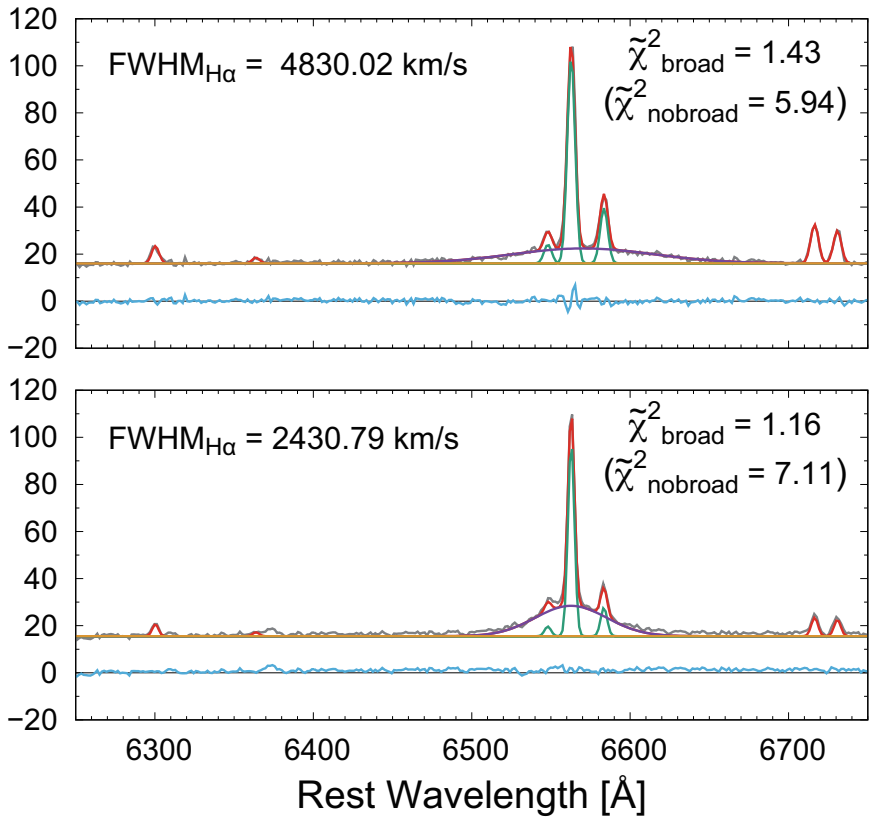

tion $\left(\sim 8.7 \times 10^{4} \mathrm{~cm}^{-3}\right)$ is significantly suppressed due to the collisional de-excitation effect. On the other hand, a very high ionization parameter results in a higher relative ionic abundance of $\mathrm{N}^{2+}$ (i.e., a lower relative ionic abundance of $\mathrm{N}^{+}$), that results in a weaker $[\mathrm{N} \mathrm{II}] \lambda 6584$ emission. Therefore, in this section, we examine whether the 43 BPT-valley AGNs are characterized by a very high electron density or very high ionization parameter or not, and test whether the AGNs in the BPT-valley are characterized by low-metallicity gas or not.

\subsection{Electron density}

The emission-line flux ratios of $[\mathrm{S}$ II $] \lambda 6717 / \lambda 6731$ and $[\mathrm{O}$ II $] \lambda 3729 / \lambda 3726$ are famous good indicators of the electron density (e.g., Osterbrock 1989). In this work, we use the $[\mathrm{S}$ II] $\lambda 6717 / \lambda 6731$ line ratio to estimate electron density, because the wavelength separation of the $[\mathrm{O}$ II] doublet is too small to be well resolved with the SDSS spectral resolution. We use an IRAF routine temden for deriving the electron density from the $[\mathrm{S} \mathrm{II}] \lambda 6717 / \lambda 6731$ ratio, by assuming the electron temperature of $10,000 \mathrm{~K}$. Here we derive the electron density whose $[\mathrm{S} \mathrm{II}] \lambda 6717 / \lambda 6731$ ratio is within the range of $0.5-1.4$. No BPT-valley objects show the [S II] $\lambda 6717 / \lambda 6731$ ratio lower than 0.5 (i.e., the highdensity limit) while 11 among the 70 BPT-valley objects show the flux ratio higher than 1.4 (i.e., the low-density limit). Among 14,252 Seyfert sample, only 12 objects show the $[\mathrm{S}$ II] $\lambda 6716 / \lambda 6731$ ratio lower than 0.5 while 2,880 objects show the flux ratio higher than 1.4.

Figure 9 shows the frequency distribution of the inferred gas density for objects whose $[\mathrm{S}$ II $] \lambda 6717 / \lambda 6731$ ratio is within the range of $0.5-1.4$; i.e., $41 \mathrm{BPT}$-valley AGNs (showing a broad $\mathrm{H} \alpha$ component and/or He II emission), 59 BPT-valley objects (including objects without any AGN signatures), and 11,360 Seyfert galaxies. Here we show the histograms for both BPT-valley AGNs and BPT-valley objects, because some of BPT-valley objects without any AGN signatures could be also AGNs 
Table 2

Broad-line AGNs in the BPT valley

\begin{tabular}{|c|c|c|c|c|c|}
\hline $\begin{array}{l}\text { ID } \\
\text { (1) }\end{array}$ & $\begin{array}{c}f(\mathrm{H} \alpha)_{\mathrm{n}} \\
(2)\end{array}$ & $\begin{array}{c}f(\mathrm{H} \alpha)_{\mathrm{b}} \\
(3)\end{array}$ & $\begin{array}{c}\mathrm{FWHM}_{\mathrm{H} \alpha} \\
(4)\end{array}$ & $\underset{(5)}{\mathrm{FWHM}_{[}} \mathrm{Sl}_{\mathrm{SI}}$ & $\begin{array}{c}\mathrm{EW}_{\text {rest }}(\mathrm{H} \alpha)_{\mathrm{b}} \\
(6)\end{array}$ \\
\hline \multicolumn{6}{|c|}{ broad $\mathrm{H} \alpha$ and $\mathrm{He}$ II } \\
\hline $3 \ldots \ldots \ldots$ & 991.96 & 1834.96 & 7162.24 & 245.05 & 88.10 \\
\hline & 414.20 & 309.70 & $875.81^{1}$ & 247.31 & 27.02 \\
\hline 12 . & 524.87 & 830.66 & 1682.70 & 324.79 & 57.25 \\
\hline $13 .$. & 748.78 & 728.55 & 2033.36 & 223.68 & 72.83 \\
\hline 25 . & 607.56 & 930.70 & 1974.28 & 248.66 & 159.88 \\
\hline 34. & 2072.41 & 797.10 & 2083.37 & 377.39 & 33.06 \\
\hline $65 \ldots \ldots \ldots$ & 573.27 & 761.34 & 4830.02 & 263.52 & 44.61 \\
\hline $66 \ldots \ldots \ldots$ & 448.38 & 768.76 & 2430.79 & 221.86 & 47.03 \\
\hline \multicolumn{6}{|c|}{ broad $\mathrm{H} \alpha$ and noHe II } \\
\hline $17 \ldots \ldots \ldots$ & 719.07 & 392.29 & 3396.57 & 270.94 & 44.74 \\
\hline $21 \ldots \ldots \ldots$ & 1014.89 & 1145.11 & 2440.19 & 337.97 & 44.13 \\
\hline $47 .$. & 515.66 & 726.01 & 3569.90 & 273.99 & 246.77 \\
\hline $58 .$. & 390.35 & 801.51 & 4347.42 & 307.51 & 28.83 \\
\hline $67 \ldots \ldots \ldots$ & 276.51 & 335.10 & 2372.32 & 276.41 & 25.14 \\
\hline
\end{tabular}

Note. - Col. (1): Identification number assigned in this paper. Col. (2): Flux of the nallow component of $\mathrm{H} \alpha$ in units of $10^{-17} \mathrm{erg} \mathrm{s}^{-1} \mathrm{~cm}^{-2}$. Col. (3): Flux of the broad component of $\mathrm{H} \alpha$ in units of $10^{-17} \mathrm{erg} \mathrm{s}^{-1} \mathrm{~cm}^{-2}$. Col. (4): FWHM of the broad component of $\mathrm{H} \alpha$ in units of $\mathrm{km} \mathrm{s}^{-1}$. Col. (5): FWHM of the [S II] $\lambda 6717$ (i.e., narrow component) in units of $\mathrm{km} \mathrm{s}^{-1}$. Col. (6): Rest-frame equivalent width of the broad component of $\mathrm{H} \alpha$ in units of $\AA$.

1 Classified as an object with a broad $\mathrm{H} \alpha$ component through the FWHM of the additional $\mathrm{H} \alpha$ component is less than $1000 \mathrm{~km} \mathrm{~s}^{-1}$ (see the main text).

Table 3

Classification result of the BPT-valley sample

\begin{tabular}{lcc}
\hline \hline & broad & nobroad \\
\hline He II & 8 & 30 \\
noHe II & 5 & 27 \\
\hline
\end{tabular}

(see Section 3.3). The median density of the BPT-valley AGNs, BPT-valley objects, and Seyfert galaxies are 210 $\mathrm{cm}^{-3}, 210 \mathrm{~cm}^{-3}$, and $270 \mathrm{~cm}^{-3}$, respectively. In order to investigate whether the frequency distribution of the gas density is statistically different among the samples, we apply the Kolmogorov-Smirnov (K-S) statistical test with a null hypothesis that the frequency distribution of the gas density of two classes of objects comes from the same underlying population. The derived K-S probability for the BPT-valley AGNs and Seyferts is 0.207, while that for the BPT-valley objects and Seyferts is 0.146. These results strongly suggest that the BPT-valley sample is not characterized by the higher gas density with respect to the Seyfert sample.

\subsection{Ionization parameter}

The ionization palameter is the ratio of the number density of hydrogen-ionizing photons to that of Hydrogen atoms. In order to investigate the ionization parameter, the $[\mathrm{O}$ III $] \lambda 5007 /[\mathrm{O}$ II $] \lambda 3727$ flux ratio is a useful indicator because this ratio does not suffer significantly from chemical properties of the gas in both AGNs and star-forming galaxies (see, e.g., Komossa \& Schulz 1997; Nagao et al. 2002; Nakajima \& Ouchi 2014). Note that this flux ratio is sensitive also to the gas density if the density is higher than the critical density of $[\mathrm{O}$ II $]\left(\sim 10^{3.5}\right.$ $\mathrm{cm}^{-3}$ ), but the typical density of NLRs inferred from the [S II] doublet ratio is much lower than that as described in Section 4.1. Though the dust reddening is not corrected to study the BPT diagram due to the small wavelength separation of emission-line pairs used for the BPT diagram (Section 2), we should correct for the reddening effect to investigate the $[\mathrm{O}$ III $] \lambda 5007 /[\mathrm{O}$ II $] \lambda 3727$ flux ratio. For this correction, we assume $R_{V}=A_{V} / E(B-V)=3.1$ and the intrinsic flux ratio of $\mathrm{H} \alpha \lambda 6584 / \mathrm{H} \beta \lambda 4861=3.1$, and adopt the reddening curve of Cardelli et al. (1989).

Figure [10 shows the histogram of the [O III] $\lambda 5007 /$ [O II] $] \lambda 3727$ line ratio of the BPT-valley AGNs, BPTvalley objects, and Seyferts, with $\mathrm{S} / \mathrm{N}\left(\left[\begin{array}{ll}\mathrm{O} & \mathrm{II}\end{array}\right] \lambda 3727\right)>$ 3 . Here it should be noted that the BPT-valley objects show $\log ([\mathrm{O}$ III $] \lambda 5007 / \mathrm{H} \beta \lambda 4861)>0.5$ by definition, while the Seyfert galaxies could have much lower $[\mathrm{O}$ III $] \lambda 5007 / \mathrm{H} \beta \lambda 4861$ flux ratios down to $\sim-0.2$. This may introduce a selection effect in the sense that strong [O III] emitters could be selectively included in the BPTvalley sample. Therefore, for reducing this selection effect, only objects with $\log ([\mathrm{O}$ III $] \lambda 5007 / \mathrm{H} \beta \lambda 4861)>0.5$ are examined for assessing the ionization parameter. After adopting this additional criterion, the numbers of the BPT-valley AGNs, BPT-valley objects, and Seyferts examined in Figure 10 are 42, 69, and 8,500, respectively. This figure shows that the BPT-valley samples seem to show systematically higher $[\mathrm{O}$ III $] \lambda 5007 /[\mathrm{O}$ II $] \lambda 3727$ flux ratios than the Seyfert sample. The median values of the logarithmic [O III $] \lambda 5007 /[\mathrm{O}$ II $] \lambda 3727$ flux ratios of the BPT-valley AGNs, BPT-valley objects, and Seyferts are $0.67,0.65$, and 0.46 , respectively. In order to investigate whether or not the distributions of the $[\mathrm{O}$ III $] \lambda 5007 /[\mathrm{O}$ II $] \lambda 3727$ line ratio are statistically different between BPT-valley sample and Seyfert sample, we apply the K-S statistical test. The K-S probability that the underlying distribution of these two distributions is the same is $3.925 \times 10^{-6}$ for the BPT-valley AGNs and Seyferts, while $1.803 \times 10^{-5}$ for the BPT-valley objects and Seyferts. These results suggest that the BPT-valley samples have statistically higher [O III $] \lambda 5007 /[\mathrm{O}$ II $] \lambda 3727$ 

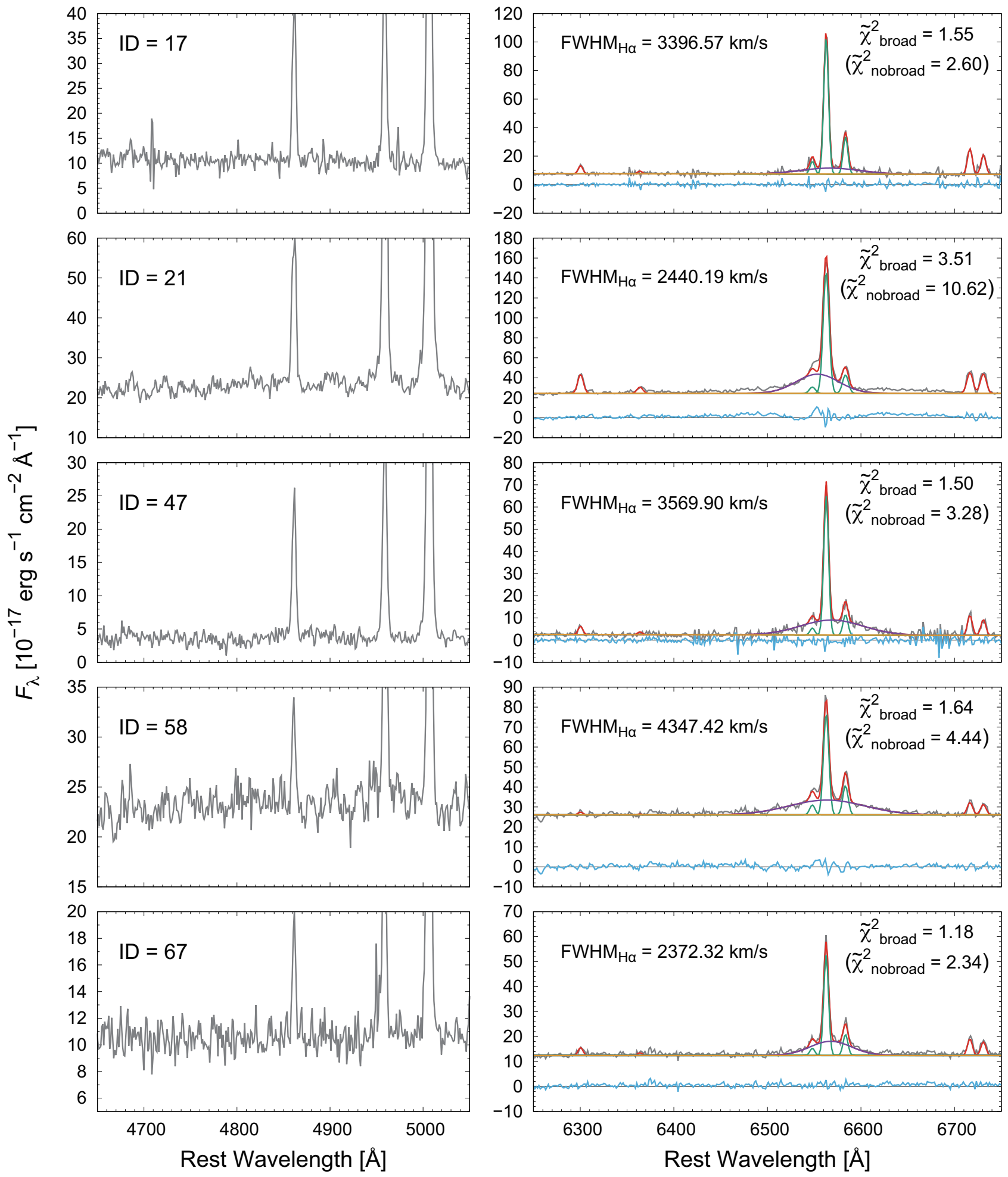

Figure 5. Same as Figure 4 but for objects showing the broad $\mathrm{H} \alpha$ emission but without the He II line. 

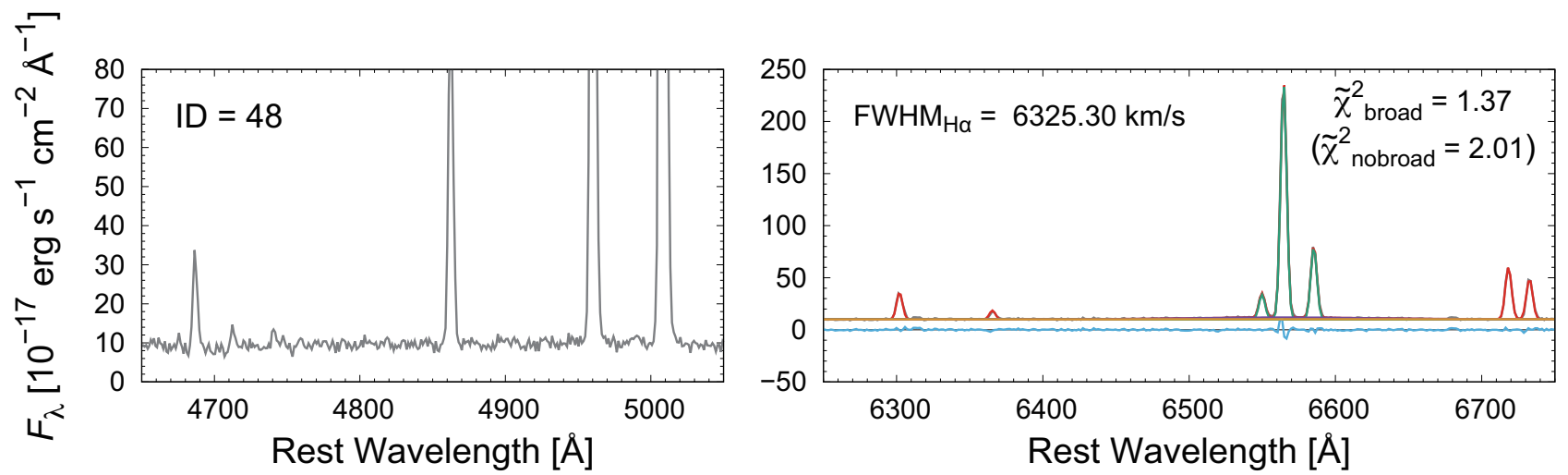

Figure 6. Same as Figure 4 but for an example of objects whose fitting result does not satisfy Equation 5

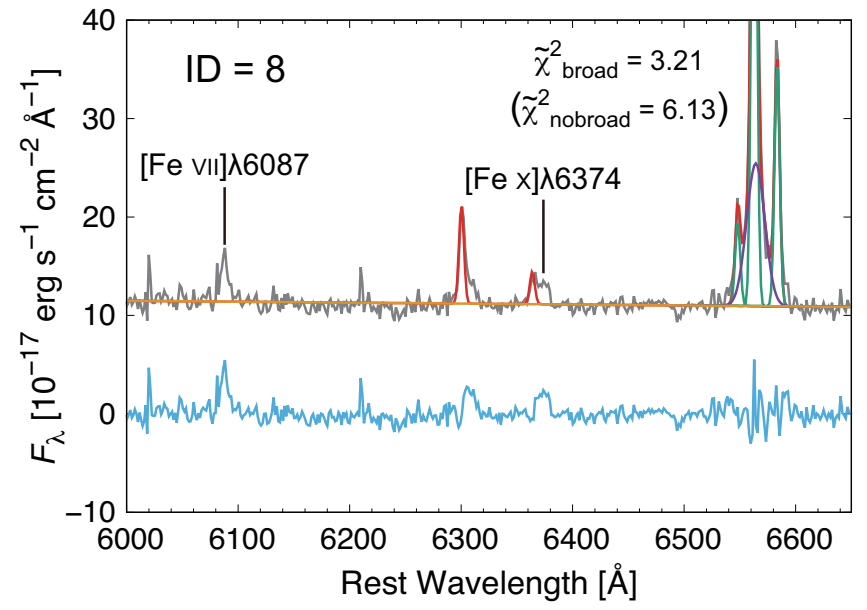

Figure 7. Same as Figure 4 but for objects ID $=8$, whose FWHM of broad $\mathrm{H} \alpha$ component is less than $1500 \mathrm{~km} \mathrm{~s}^{-1}$ (see Table 2). High-ionization forbidden emission lines of [Fe VII] $\lambda 6087$ and $[\mathrm{Fe} \mathrm{x}] \lambda 6374$ are clearly detected. Note that fitting range is from 6200 to $6800 \AA$ in the restframe. Continuum fitting is extrapolated below $6200 \AA$.

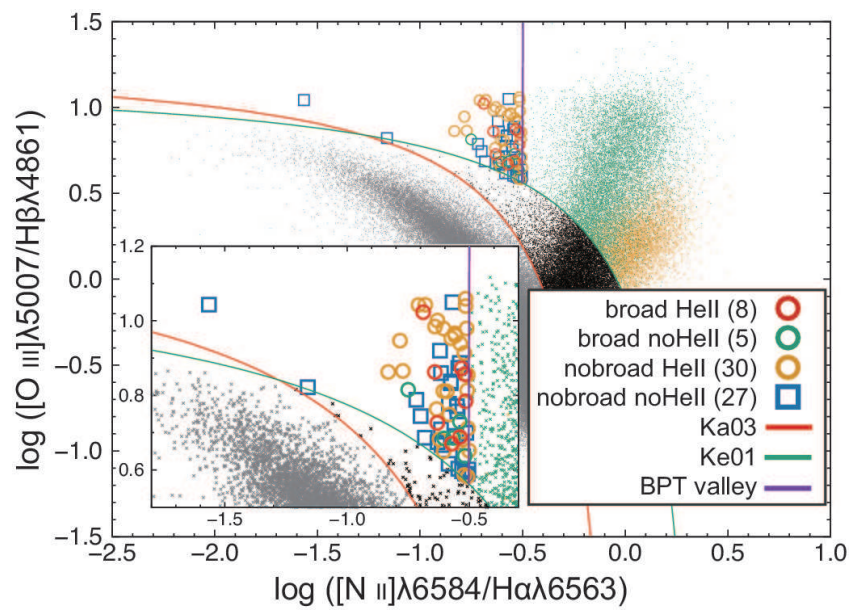

Figure 8. The BPT diagram ([N $\mathrm{NI}] \lambda 6584 / \mathrm{H} \alpha \lambda 6563$ versus $[\mathrm{O}$ III $] \lambda 5007 / \mathrm{H} \beta \lambda 4861)$ with the classification result of the BPT valley sample among the SDSS DR7 emission-line objects. The numbers of various galaxy populations are shown in the parenthesis in the lower-right box.

flux ratios, i.e., the ionization parameter, than the

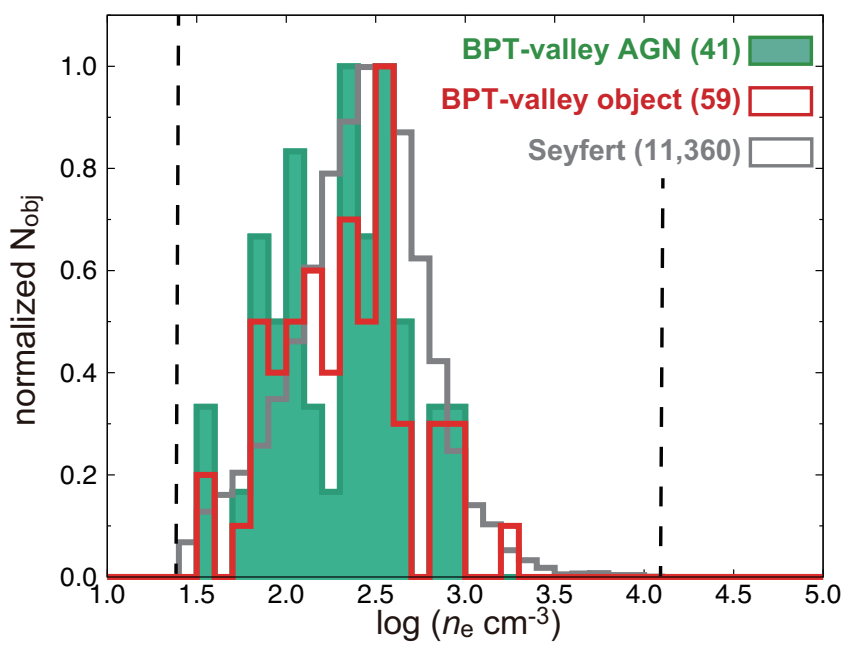

Figure 9. Histograms of the electron density of the BPT-valley AGN (filled green), BPT-valley objects (open red), and Seyfert galaxies (open gray), normalized by their peak count. Dashed lines denote the range of the electron density measurable through the [S II] doublet ratio.

Seyfert sample. Note that it is well known that lowmetallicity galaxies are generally characterized by a relatively high ionization parameter, at least for star-forming galaxies (e.g., Nagao et al. 2006a). It may be interesting that the BPT-valley objects show a clear edge at the lower side of the $[\mathrm{O}$ III $] \lambda 5007 /[\mathrm{O}$ II $] \lambda 3727$ distribution in Figure 10. However, probably this feature is not statistically significant, because the number of BPT-valley objects is not enough to discuss the tail of the frequency distribution of the $[\mathrm{O}$ III $] \lambda 5007 /[\mathrm{O}$ II $] \lambda 3727$ flux ratio.

In the next subsection, we will examine whether or not this difference in the ionization parameter can be responsible for the lower $[\mathrm{N} \mathrm{II}] \lambda 6584 / \mathrm{H} \alpha \lambda 6563$ ratio observed in the BPT-valley samples with respect to the Seyfert sample.

\subsection{Model calculations}

As shown in Section 4.2, the ionization parameter of the BPT valley sample is higher than that of the Seyfert sample. Since it is interesting to examine either the BPTvalley AGNs are characterized by a low metallicity or a high ionization parameter, we perform photoionization model calculations.

We perform photoionization model calculations for 


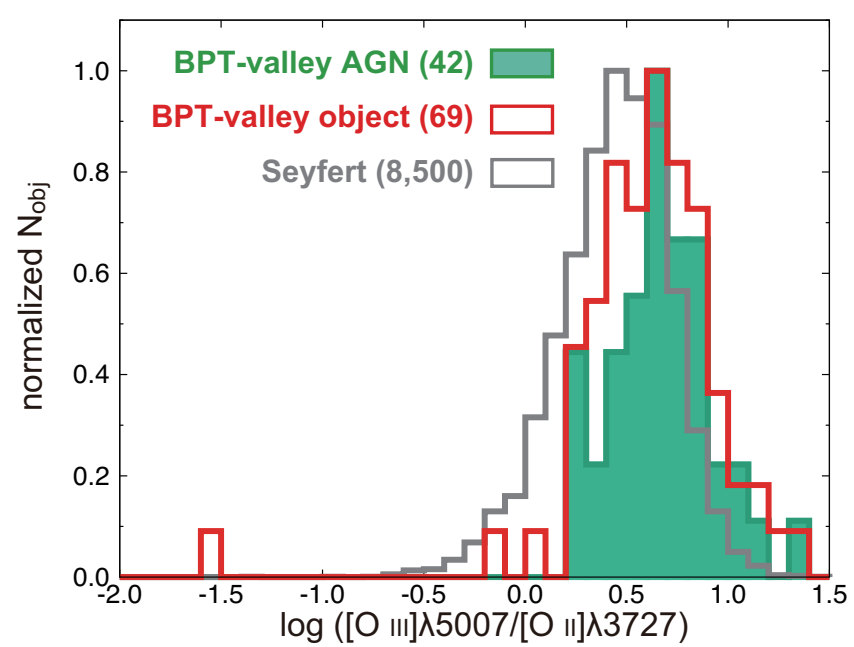

Figure 10. Same as Figure 9 but for the $[\mathrm{O}$ III $] \lambda 5007 /[\mathrm{O}$ II $] \lambda 3727$ flux ratio.

simulating the NLR of AGNs, using the code CLOUDY version 13.03 (Ferland et al. 1998). Here the main parameters for CLOUDY calculations are as follows (see Nagao et al. 2001a, for more details):

1. The hydrogen density of the cloud $\left(n_{\mathrm{H}}\right)$.

2. The ionization parameter $(U)$.

3. The chemical composition of the gas.

4. The shape of the input SED.

We calculate photoionization models covering the following ranges of parameters: $10^{1} \mathrm{~cm}^{-3} \leq n_{\mathrm{H}} \leq$ $10^{6} \mathrm{~cm}^{-3}$ and $10^{-4} \leq U \leq 10^{-1}$. We set the gasphase elemental abundance ratios to be the solar ones. The adopted solar abundances relative to hydrogen are taken from Grevesse \& Anders (1989) with extensions by Grevesse \& Noels (1993). The adopted metallicity (i.e., the solar one) is not typical for usual Seyfert galaxies (whose NLR metallicity is generally higher than the solar metallicity), possibly nor BPT-valley AGNs (that could have sub-solar metallicity). However, as described below, it is useful to fix the metallicity to examine whether the ionization parameter alone can account for the difference in the emission-line flux ratios between BPT-valley objects and Seyferts. For the input SED, we adopt the following one:

$$
f_{\nu}=\nu^{\alpha_{\mathrm{UV}}} \exp \left(-\frac{h \nu}{k T_{\mathrm{BB}}}\right) \exp \left(-\frac{k T_{\mathrm{IR}}}{h \nu}\right)+a \nu^{\alpha \mathrm{X}}
$$

as a typical spectrum of AGNs (see Ferland 1996). $k T_{\mathrm{IR}}$ is the infrared cutoff of the big-blue bump, and we adopt $k T_{\mathrm{IR}}=0.01$ ryd (see Ferland 1996). $\alpha_{\mathrm{UV}}$ is the slope of the low-energy side of the big-blue bump. We adopt $\alpha_{\mathrm{UV}}=0.5$, which is typical for AGNs (Ferland 1996). $\alpha_{\text {ox }}$ is the UV-to-X-ray spectral slope, which determines the parameter $a$ in equation (6). We adopt $\alpha_{\mathrm{ox}}=-1.35$, which is the average value of nearby Seyfert 1 galaxies (see Walter \& Fink 1993). $\alpha_{\mathrm{x}}$ is the X-ray slope, and we adopt $\alpha_{\mathrm{x}}=-0.85$ (see Nagao et al. 2001a). $T_{\mathrm{BB}}$ is the characterizing the shape of the big-blue bump, and we adopt 490,000 K (see Nagao et al. 2001a). The calculations end at the depth where the temperature falls to $3,000 \mathrm{~K}$, below which gas does not contribute significantly to the flux of optical emission lines.

Figure 11 shows the results of the photoionization model calculations, overlaid on the BPT diagram. Though the density effect is not significant in the range of $10^{1} \mathrm{~cm}^{-3}<n_{\mathrm{H}}<10^{5} \mathrm{~cm}^{-3}$, we can see the effect of the collisional de-excitation at $n_{\mathrm{H}}>10^{4} \mathrm{~cm}^{-3}$. However, this figure suggests that the difference in the $[\mathrm{N}$ II] $\lambda 6584 / \mathrm{H} \alpha \lambda 6583$ flux ratio is more easily explained by the difference in the ionization parameter rather than by the difference in the gas density. More specifically, a higher ionization parameter by $0.5-1$ dex in the BPTvalley objects with respect to the Seyfert sample is required to explain the lower [N II] $\lambda 6584 / \mathrm{H} \alpha \lambda 6583$ flux ratio of the BPT-valley objects.

For examining whether the BPT-valley objects have a higher ionization parameter than the Seyfert sample, we investigate another diagnostic diagram that consists of the emission-line flux ratios of $[\mathrm{O}$ III $] \lambda 5007 /[\mathrm{O}$ II $] \lambda 3727$ and $[\mathrm{O}$ I $] \lambda 6300 /[\mathrm{O}$ III $] \lambda 5007$ (Figure [12). This diagram is useful to examine the effect of ionization parameter without suffering from the metallicity effect, because only oxygen lines are used and thus less sensitive to the metallicity. Figure 12 shows that the BPT-valley sample and Seyfert sample have a similar gas density, that is consistent with our analysis presented in Section 4.1. More interestingly, Figure 12 shows that the BPT-valley sample shows a systematically higher ionization parameter than the Seyfert sample, but the inferred difference in the ionization parameters is only less than 0.5 dex. This strongly suggests that the lower $[\mathrm{N}$ II $] \lambda 6584 / \mathrm{H} \alpha \lambda 6563$ flux ratio in the BPT-valley sample with respect to the Seyfert sample is not explained by the ionization parameter (nor the gas density, as described in Section 4.1). Therefore we conclude that the BPT-valley AGNs are characterized by a systematlcally lower metallicity than the Seyfert sample, as originally proposed by Groves et al. (2006).

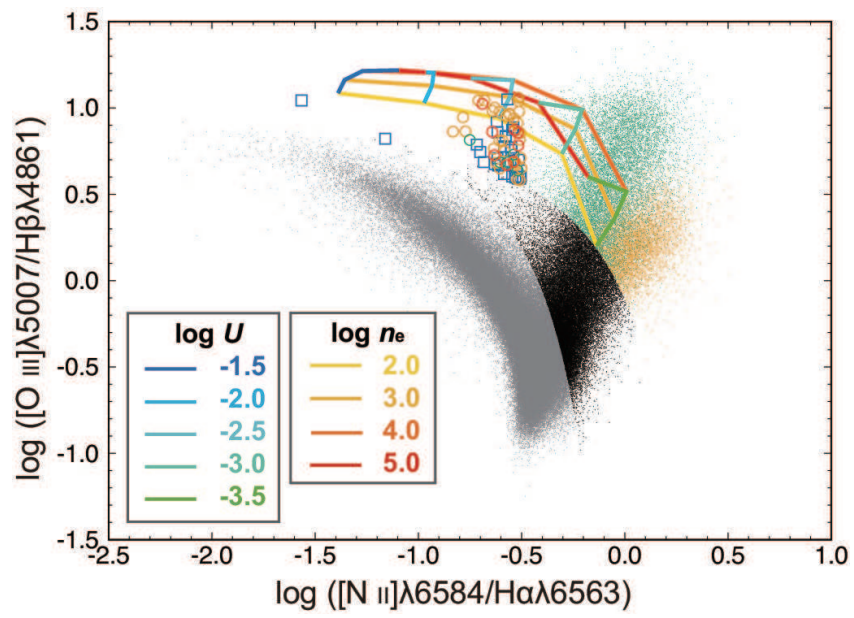

Figure 11. Same as Figure 8 (without the inset panel in Figure 8 but grids of photoionization models are overlaid. Different colors of lines denote different parameters adopted in the calculations, as shown in the inset panels.

\section{DISCCUSIONS}




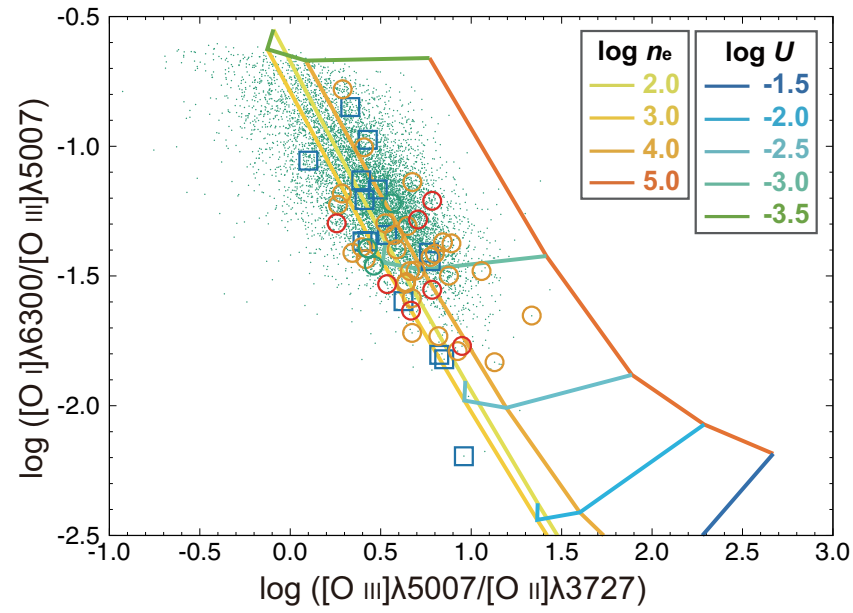

Figure 12. Diagnostic diagram of $[\mathrm{O}$ III $] \lambda 5007 /[\mathrm{O}$ II $] \lambda 3727$ versus $[\mathrm{O}$ I $] \lambda 6300 /[\mathrm{O}$ III $] \lambda 5007$. The symbols and lines are the same as in Figure 11 Note that only the BPT valley objects with $\mathrm{S} / \mathrm{N}>5$ of the $[\mathrm{O}$ II] $\lambda 33727,[\mathrm{O}$ III] $\lambda 5007$ and $[\mathrm{O}$ I] $\lambda 6300$ line are plotted.

As mentioned Section 1, low-metallicity AGNs are interesting to study the early phase of the AGN evolution. However low-metallicity AGNs are very rare, so that little has been reported on physical property of lowmetallicity AGNs. In this section, we present some basic properties of BPT-valley objects which are expected to be low-metallicity AGNs.

\subsection{Stellar mass}

Naively it is expected that the stellar mass of lowmetallicity AGNs is expected to be relatively low, as suggested by the mass-metallicity relation seen in starforming galaxies (e.g., Tremonti et al. 2004; Lee et al. 2006). Accordingly Groves et al. (2006) introduced a mass criterion (i.e., $M_{*}<10^{10} \mathrm{M}_{\odot}$ ) to select lowmetallicity AGNs. However, it is not clarified whether low-metallicity AGNs should be always found in a sample of AGNs with a low-mass host galaxy. Therefore, in this paper, we select low-metallicity AGNs without stellar-mass cut and investigate the mass distribution of host galaxies of low-metallicity AGNs. Here the stellar mass has been measured and given in the MPA-JHU DR7 catalog (see also Kauffmann et al. 2003a). Among the 43 BPT-valley AGNs and 70 BPT-valley objects, the host mass is available for 39 and 64 objects, respectively. Figure 13 shows the histogram of the stellar mass of the 39 BPT-valley AGNs, 64 BPT-valley objects and 13,662 Seyferts. The median of the stellar mass of the BPT-valley AGNs, BPT-valley objects and Seyferts are $10^{10.15} \mathrm{M}_{\odot}, 10^{10.07} \mathrm{M}_{\odot}$ and $10^{10.77} \mathrm{M}_{\odot}$, respectively. This result clearly shows that the stellar mass of the BPT-valley AGNs is systematically lower than that of Seyferts. However, interestingly, a substantial fraction of the BPT-valley AGN (23 among 39 objects) are actually hosted by galaxies with $M_{*}>10^{10} \mathrm{M}_{\odot}$, suggesting that low-metallicity AGNs are not necessarily hosted by lowmass galaxies. Note that such low-metallicity AGNs with a relatively massive host galaxy cannot be selected by the criteria of Groves et al. (2006) due to the mass criterion of $M_{*}<10^{10} \mathrm{M}_{\odot}$. Such low-metallicity AGNs hosted by a relatively massive host galaxy may be realized by taking into account of the inflow of low-metallicity gas from the surrounding environment (e.g., Husemann et al. 2011).

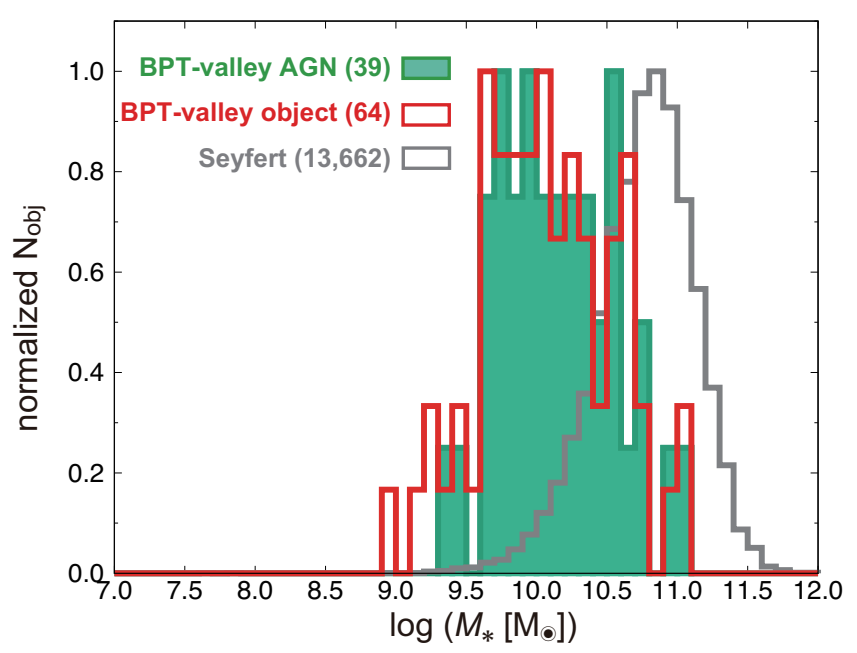

Figure 13. Same as Figure 9 but for the stellar mass.

\subsection{Electron temperature}

Considering the effect of the metal cooling, lowmetallicity AGNs are expected to be characterized by the higher electron temperature. Hence we investigate the $\left[\begin{array}{ll}\mathrm{O} & \mathrm{III}\end{array}\right] \lambda \lambda(4959+5007) /[\mathrm{O} \mathrm{III}] \lambda 4363$ line ratio which is very sensitive to the gas temperature. Here it should be mentioned that, [O III] $\lambda \lambda(4959+$ $5007) /[\mathrm{O}$ III $] \lambda 4363$ line ratio also depends on the electron density (see, e.g., Nagao et al. 2001b). Therefore we investigate the $[\mathrm{O}$ III $] \lambda \lambda(4959+5007) /[\mathrm{O}$ III $] \lambda 4363$ and $[\mathrm{S}$ II $] \lambda 6717 /[\mathrm{S}$ II $] \lambda 6731$ line ratios simultaneously in Figure [14. Here this figure shows the emission-line flux ratios of BPT-valley objects and Seyferts but only for objects with a significant detection of the $[\mathrm{O}$ III $] \lambda 4363$ line $(\mathrm{S} / \mathrm{N}>3)$. As described in Section 4.2 , only objects with $\log ([\mathrm{O}$ III $] \lambda 5007 / \mathrm{H} \beta)>0.5$ are used (that results in 9,043 Seyferts and 70 BPT-valley objects). Note that $[\mathrm{O}$ III $] \lambda \lambda(4959+5007) /[\mathrm{O}$ III $] \lambda 4363$ line ratio is corrected for the reddening effect in the same way as Section 4.2. The median values of $\log ([\mathrm{S}$ II $] \lambda 6717 /[\mathrm{S}$ II $] \lambda 6731)$ of the BPT-valley AGNs, BPT-valley objects and Seyferts with a $[\mathrm{O}$ III $] \lambda 4363$ detection are $0.088,0.088$ and 0.055 , respectively. Therefore the electron density of the BPTvalley sample is slightly higher than that of Seyferts as already mentioned in Section 4.1. The median of log $([\mathrm{O}$ III $] \lambda \lambda(4959+5007) /[\mathrm{O}$ III $] \lambda 4363)$ of the BPT-valley AGN, BPT-valley objects and Seyferts are 1.77, 1.77 and 1.79, respectively. This result suggests that the electron temperature of the BPT-valley objects is not significantly higher than that of Seyferts. However, the fraction of objects showing a significant $(\mathrm{S} / \mathrm{N}>3)[\mathrm{O}$ III $] \lambda 4363$ emission is very different between the Seyferts and BPTvalley objects. More specifically, 44 among the $70 \mathrm{BPT}$ valley objects $(\sim 63 \%)$ show the $[\mathrm{O}$ II $] \lambda 4363$ emission while only 1,516 among 9,043 Seyferts $(\sim 17 \%)$ show the $[\mathrm{O}$ III $] \lambda 4363$ line. This difference infers that generally the gas temperature of the NLR in BPT-velley objects tends to be so high that the [O III] $\lambda 4363$ line is detected in most cases, while the typical gas temperature of the NLR in Seyferts may be lower than that in 
BPT-valley objects and only highly biased objects with a relatively high temperature in the Seyfert sample show the [O III $\lambda 4363$ line. This result is consistent to our expectation that the BPT-valley objects is actually characterized by a relatively high gas temperature, due to the low gas metallicity.

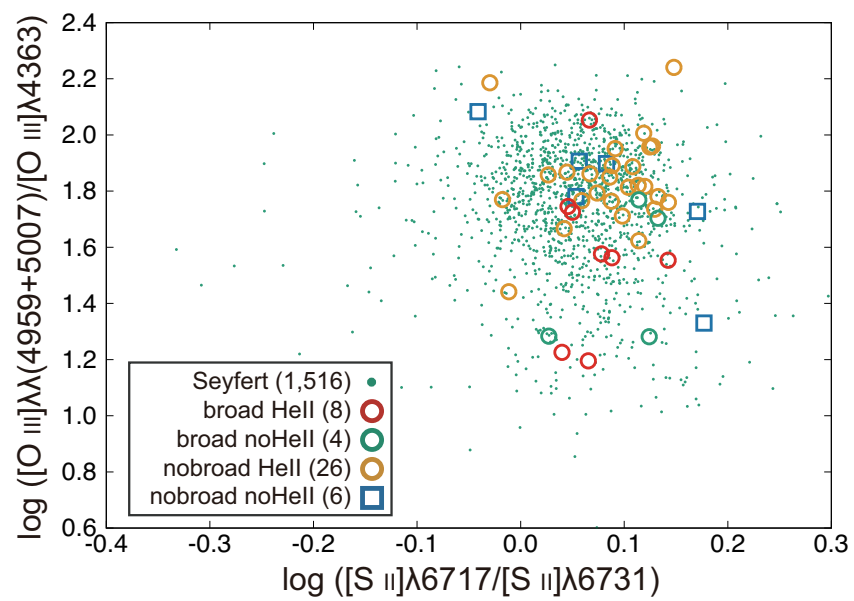

Figure 14. Diagnostic diagram of $\left[\begin{array}{ll}\mathrm{O} & \mathrm{III}\end{array}\right] \lambda \lambda(4959+5007) /$ $[\mathrm{O}$ III $] \lambda 4363$ versus $[\mathrm{S} \mathrm{II}] \lambda 6717 /[\mathrm{S}$ II $] \lambda 6731$. The symbols are the same as in Figure 8 Note that only the BPT valley objects with $\mathrm{S} / \mathrm{N}>3$ of the $[\mathrm{O} \mathrm{III}] \lambda 4363$ line are plotted.

\section{CONCLUSIONS}

In this paper, we focus on low-metallicity $\operatorname{AGNs}\left(Z_{\mathrm{NLR}}\right.$ $\left.\lesssim 1 Z_{\odot}\right)$ which are very rare but important since they are in the early phase of the galaxy-SMBH co-evolution. Specifically, in this work it is examined whether the BPTvalley selection is an effective and reliable way to identify low-metallicity AGNs, as proposed by Groves et al. (2006). The main results are as follows:

- We select 70 BPT valley sample which expected low metallicity AGN from 14,253 Seyfert galaxies of MPA-JHU SDSS DR7 galaxy catalog.

- Out of 70 BPT-valley objects, 43 objects show clear evidence of the AGN based on the detection of the broad $\mathrm{H} \alpha$ component and/or He II $\lambda 4686$ emission.

- The typical gas density of the BPT-valley sample $\left(\sim 210 \mathrm{~cm}^{-3}\right)$ is not higher than that of the Seyfert sample $\left(\sim 270 \mathrm{~cm}^{-3}\right)$, suggesting that the lower $[\mathrm{N}$ II] $\lambda 6584 / \mathrm{H} \alpha \lambda 6563$ ratio in the BPT-valley AGNs with respect to the Seyfert sample is not caused by the collisional de-excitation effect.

- The higher $[\mathrm{O}$ III $] \lambda 5007 /[\mathrm{O}$ II $] \lambda 3727$ ratio in the BPT-valley sample $(\sim 4.5)$ with respect to that in the Seyfert sample $(\sim 2.9)$ suggests a typically higher ionization parameter of the BPT-valley sample; however, photoionization models suggest that the inferred difference in the ionization parameter between the BPT-valley sample and Seyfert sample is not enough to explain the observed lower $[\mathrm{N}$ II] $\lambda 6584 / \mathrm{H} \alpha \lambda 6563$ ratio of the BPT-valley sample.
- The BPT-valley selection for identifying lowmetallicity AGNs is thus confirmed to be a useful method; in our analysis, more than $60 \%$ of the BPT-valley sample are low-metallicity AGNs $\left(Z_{\mathrm{NLR}} \lesssim 1 Z_{\odot}\right)$.

We would like to thank the anonymous referee for her/his careful reading this paper and useful suggestions, and also Masaru Kajisawa and Kazuyuki Ogura for their useful comments. TN is financially supported by JSPS grants Nos. 25707010, 16H01101, and 16H03958. KM is also supported by JSPS grant No. 14J01811. Funding for the SDSS and SDSS-II has been provided by the Alfred P. Sloan Foundation, the Participating Institutions, the National Science Foundation, the U.S. Department of Energy, the National Aeronautics and Space Administration, the Japanese Monbukagakusho, the Max Planck Society, and the Higher Education Funding Council for England. The SDSS Web Site is http://www.sdss.org/. The SDSS is managed by the Astrophysical Research Consortium for the Participating Institutions. The Participating Institutions are the American Museum of Natural History, Astrophysical Institute Potsdam, University of Basel, University of Cambridge, Case Western Reserve University, University of Chicago, Drexel University, Fermilab, the Institute for Advanced Study, the Japan Participation Group, Johns Hopkins University, the Joint Institute for Nuclear Astrophysics, the Kavli Institute for Particle Astrophysics and Cosmology, the Korean Scientist Group, the Chinese Academy of Sciences (LAMOST), Los Alamos National Laboratory, the Max-Planck-Institute for Astronomy (MPIA), the MaxPlanck-Institute for Astrophysics (MPA), New Mexico State University, Ohio State University, University of Pittsburgh, University of Portsmouth, Princeton University, the United States Naval Observatory, and the University of Washington.

\section{REFERENCES}

Abazajian, K. N., Adelman-McCarthy, J. K., Agüeros, M. A., et al. 2009, ApJS, 182, 543

Baldwin, J. A., Phillips, M. M., \& Terlevich, R. 1981, PASP, 93, 5 Cardelli, J. A., Clayton, G. C., \& Mathis, J. S. 1989, ApJ, 345, 245

Carniani, S., Marconi, A., Biggs, A., et al. 2013, A\&A, 559, A29

Crenshaw, D. M., \& Kraemer, S. B. 2000, ApJ, 532, L101

Ferland, G. J. 1996, Hazy, A Brief Introduction to Cloudy 90

Ferland, G. J., Korista, K. T., Verner, D. A., et al. 1998, PASP, 110,761

Grevesse, N., \& Anders, E. 1989, in American Institute of Physics Conference Series, Vol. 183, Cosmic Abundances of Matter, ed. C. J. Waddington, 1-8

Grevesse, N., \& Noels, A. 1993, in Origin and Evolution of the Elements, ed. N. Prantzos, E. Vangioni-Flam, \& M. Casse, $15-25$

Groves, B. A., Heckman, T. M., \& Kauffmann, G. 2006, MNRAS, 371,1559

Hayashi, M., Ly, C., Shimasaku, K., et al. 2015, PASJ, 67, 80

Heckman, T. M. 1980, A\&A, 87, 152

Heckman, T. M., Miley, G. K., \& Green, R. F. 1984, ApJ, 281 525

Ho, L. C., Filippenko, A. V., \& Sargent, W. L. W. 1997, ApJS, 112,315

Husemann, B., Wisotzki, L., Jahnke, K., \& Sánchez, S. F. 2011, A\&A, 535, A72

Izotov, Y. I., \& Thuan, T. X. 2008, ApJ, 687, 133

Kauffmann, G., Heckman, T. M., White, S. D. M., et al. 2003a, MNRAS, 341,33

Kauffmann, G., Heckman, T. M., Tremonti, C., et al. 2003b,

MNRAS, 346, 1055 
Kawakatu, N., Umemura, M., \& Mori, M. 2003, ApJ, 583, 85 Kewley, L. J., Dopita, M. A., Leitherer, C., et al. 2013, ApJ, 774, 100

Kewley, L. J., Dopita, M. A., Sutherland, R. S., Heisler, C. A., \& Trevena, J. 2001, ApJ, 556, 121

Kewley, L. J., Groves, B., Kauffmann, G., \& Heckman, T. 2006 MNRAS, 372, 961

Kojima, T., Ouchi, M., Nakajima, K., et al. 2016, ArXiv e-prints, arXiv:1605.03436

Komossa, S., \& Schulz, H. 1997, A\&A, 323, 31

Kormendy, J., \& Ho, L. C. 2013, ARA\&A, 51, 511

Kriss, G. 1994, in Astronomical Society of the Pacific Conference Series, Vol. 61, Astronomical Data Analysis Software and Systems III, ed. D. R. Crabtree, R. J. Hanisch, \& J. Barnes, 437

Lamastra, A., Menci, N., Maiolino, R., Fiore, F., \& Merloni, A. 2010, MNRAS, 405, 29

Lee, H., Skillman, E. D., Cannon, J. M., et al. 2006, ApJ, 647, 970

Magorrian, J., Tremaine, S., Richstone, D., et al. 1998, AJ, 115, 2285

Marconi, A., \& Hunt, L. K. 2003, Ap.J, 589, L21

Masters, D., McCarthy, P., Siana, B., et al. 2014, ApJ, 785, 153

Matsuoka, K., Nagao, T., Maiolino, R., Marconi, A., \& Taniguchi, Y. 2009, A\&A, 503, 721

Mendoza, C. 1983, in IAU Symposium, Vol. 103, Planetary Nebulae, ed. D. R. Flower, 143-172

Murayama, T., \& Taniguchi, Y. 1998, ApJ, 497, L9

Nagao, T., Maiolino, R., \& Marconi, A. 2006a, A\&A, 459, 85

Nagao, T., Marconi, A., \& Maiolino, R. 2006b, A\&A, 447, 157
Nagao, T., Murayama, T., Shioya, Y., \& Taniguchi, Y. 2002, ApJ, 567,73

Nagao, T., Murayama, T., \& Taniguchi, Y. 2001a, ApJ, 546, 744 -. 2001b, ApJ, 549, 155

Nagao, T., Taniguchi, Y., \& Murayama, T. 2000, AJ, 119, 2605

Nakajima, K., \& Ouchi, M. 2014, MNRAS, 442, 900

Newman, S. F., Buschkamp, P., Genzel, R., et al. 2014, ApJ, 781, 21

Osterbrock, D. E. 1989, Astrophysics of gaseous nebulae and active galactic nuclei (Mill Valley: University Science Books)

Reines, A. E., \& Volonteri, M. 2015, ApJ, 813, 82

Schramm, M., Wisotzki, L., \& Jahnke, K. 2008, A\&A, 478, 311

Schulze, A., \& Wisotzki, L. 2011, A\&A, 535, A87

Shapley, A. E., Reddy, N. A., Kriek, M., et al. 2015, ApJ, 801, 88

Shirazi, M., Brinchmann, J., \& Rahmati, A. 2014, ApJ, 787, 120

Steidel, C. C., Rudie, G. C., Strom, A. L., et al. 2014, ApJ, 795, 165

Tremonti, C. A., Heckman, T. M., Kauffmann, G., et al. 2004, ApJ, 613, 898

van Zee, L., Salzer, J. J., \& Haynes, M. P. 1998, ApJ, 497, L1

Vanden Berk, D. E., Richards, G. T., Bauer, A., et al. 2001, AJ, 122,549

Walter, R., \& Fink, H. H. 1993, A\&A, 274, 105

Wang, R., Carilli, C. L., Neri, R., et al. 2010, ApJ, 714, 699

Yabe, K., Ohta, K., Akiyama, M., et al. 2015, PASJ, 67, 102

York, D. G., Adelman, J., Anderson, Jr., J. E., et al. 2000, AJ, 120,1579 